\title{
RICCATI EQUATIONS
}

\author{
LLOYD K. WILLIAMS \\ Department of Mathematics \\ Texas Southern University \\ Houston, Texas 77004 \\ (Received February 2, 1986)
}

ABSTRACT. In this paper we find closed form solutions of some Riccati equations. Attention is restricted to the scalar as opposed to the matrix case. However, the ones considered have important applications to mathematics and the sciences, mostly in the form of the linear second-order ordinary differential equations which are solved herewith.

KEY WORDS AND PHRASES. Riccati equations, Bäcklund transformations, Bernoulli equations. 1980 AMS SUBJECT CLASSIFICATION CODE. 34A05

1. INTRODUCTION.

The equations investigated here have a long, full history that extends well over two and a half centuries. They are closely related to second order linear equations, for example, which have an interesting history in their own right.

Attention will be focused on systems of equations and the use of various transformations of Bäcklund type. There will generally be available unknown functions which will be able to specify to suit our purposes. Afterwards, we must solve to determine functions of a different type. We must carefully distinguish the two classes of functions.

2. THE RICCATI EQUATION.

First consider the linear second order differential equation

$$
z^{\prime \prime}=A z^{\prime}+B z
$$

and note that if the function $w$ is defined by

$$
z^{\prime}=w z
$$

we get the Riccati equation

$$
w^{2}+w^{2}-A w-B=0
$$

for w. On the other hand (and in the opposite direction) the substitution

$$
y=\frac{A v}{v^{\prime}}, \quad A \neq 0
$$

in the Riccati equation

$$
y^{\prime}=y^{2}+A, \quad A \neq 0 \quad \text { (a trivial exception) }
$$

leads to the second order linear equation

$$
v^{\prime \prime}=\frac{A^{\prime}}{A} v^{\prime}+A v=0
$$


for $v$.

There is thus seen to be an equivalence of sorts between the two kinds of equations.

We proceed now to the solution of $(2.5)$. That (2.3) is subsumed in the process is readily shown by making the substitution

$$
v=P w
$$

with

$$
P^{\prime}=A P
$$

Consider the system

$$
\left(\begin{array}{c}
y^{\prime}=f u+g v \\
y=u
\end{array}\right)
$$

for unknown functions $u$ and $v$ where we may choose functions $f$ and $g$ at our convenience.

From (2.5) we have

$$
\left(\frac{f \pm \sqrt{f^{2}+4 g v-4 A}}{2}\right)^{\prime}=\frac{\left(f^{2}+2 g v\right) \pm f \sqrt{f^{2}+4 g v-4 A}}{2}
$$

Now the Bernoulli equation

$$
y^{\prime}=R y^{2}+S y
$$

is solved by quadratures for arbitrary functions $R$ and $S$. We propose to bring (2.9), or at least to derive such an equation.

Let

$$
v=w^{2}+B w+C
$$

for a new unknown, w. Once again, B,C will serve as convenient parameters. Choose $B$ so that the radicand in (2.10) will be a perfect square in $w$, that is we impose the condition:

$$
(4 g B)^{2}-4(4 g)\left(4 g C+f^{2}-4 A\right)=0, \quad g \neq 0 .
$$

Further we require that then the $w$-free inside the parenthesis on the left in (2.10) be a constant, a; and that the w-free term on the right be zero. This will give us the following Bernoulli equation for $w$ :

$$
(\sqrt{g} w)^{\prime}=g w^{2}+(g B \pm f \sqrt{g}) w=0
$$

Interpreting the conditions imposed we have

$$
\frac{f \pm \sqrt{f^{2}+4 g C-4 A}}{2}=a, \frac{\left(f^{2}+2 g C\right) \pm f \sqrt{f^{2}+4 g C-4 A}}{2}
$$

From the second of these we have

$$
\mp f \sqrt{f^{2}+4 g C-4 A}=f^{2}+2 g C
$$

whence squaring

$$
f=\frac{g C}{\sqrt{-A}}, \quad A \neq 0
$$


Then from the first equation

$$
\begin{array}{r}
g C=\frac{a+A}{1+\frac{a}{\sqrt{-A}}} \\
f=\frac{a^{2}+A}{\sqrt{-A}+a}
\end{array}
$$

It may need to be said that $a$ is the only constant here, A can be any non-zero function. Every Riccati equation can be cast in this form by suitable changes of variables.

3. METHOD OF SOLUTION.

Select a to be a non-zero constant and $g$ to be any non-zero function. Solve for $C$ and $f$ from equations (2.18) and (2.19).

Solve (2.13) for B then solve (2.14) for $w$. Now (2.12) yields $v$. With $f, g$ and $v$ the equation (2.9) becomes a linear first order differential equation to solve for $y$.

4. AN ALTERNATIVE DEVELOPMENT.

1. Define $u$ in terms of $v$ by $u^{2}+A=f u+g v$

2. Assume that equation (2.10) holds, then $u$ is a solution of (2.5) regardless of the functions $f, g$.

ACKNOWLEDGEMENT: The author wishes to thank Albert Lee and others who carefully read the manuscript.

DEDICATION: Dedicated to the Bernoulli family who began this in 1704 .

\section{REFERENCES}

1. KAMKE, E. Differentialgleichungen, Lösungs Methoden und Lösungungen, Akademischer Verlag, Leipzig, 1959 (6th ed.).

2. REID, William T. Riccati Differential Equations, Academic Press, New York, 1972.

3. DAVIS, Harold T. Introduction to Non-Linear Differential and Integral Equations, Dover, New York, 1962 .

4. BERNOVLLI, Daniel. Solutio Problematis Riccatiani Proporeti, Act. Lipo. Suppl., TOMvii 73 Acta Erudetorum 1925, 473-475.

5. GLAISHER, J.W.L. On Riccati's Equation and its Transformations and Some Definite Integrals which satisfy them, Phil. Trans. of Royal Soc. 172, 1881, 759-828.

6. LAGRANGE, R. Quelqes Théorèmes d'Integrabilite par Quadratures de 1'Equation de Riccati, Bu11. Soc. Math. de France, 66, 1938, 158-163. 


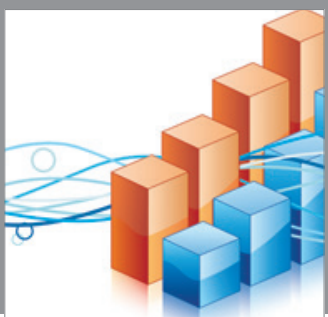

Advances in

Operations Research

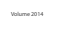

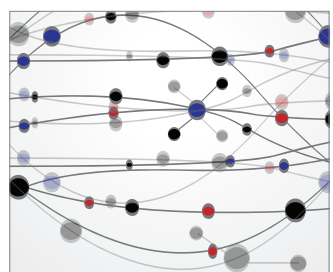

\section{The Scientific} World Journal
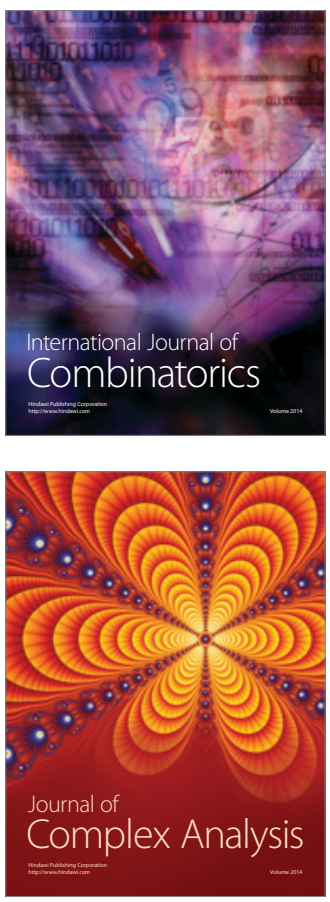

International Journal of

Mathematics and

Mathematical

Sciences
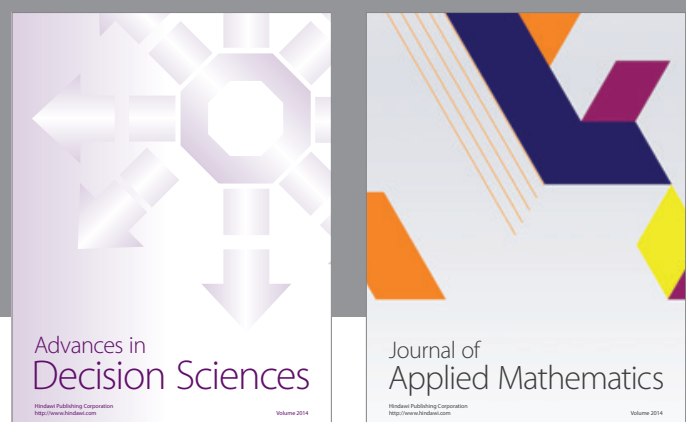

Journal of

Applied Mathematics
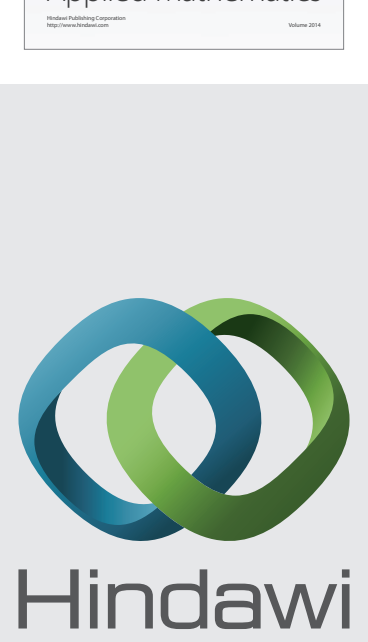

Submit your manuscripts at http://www.hindawi.com
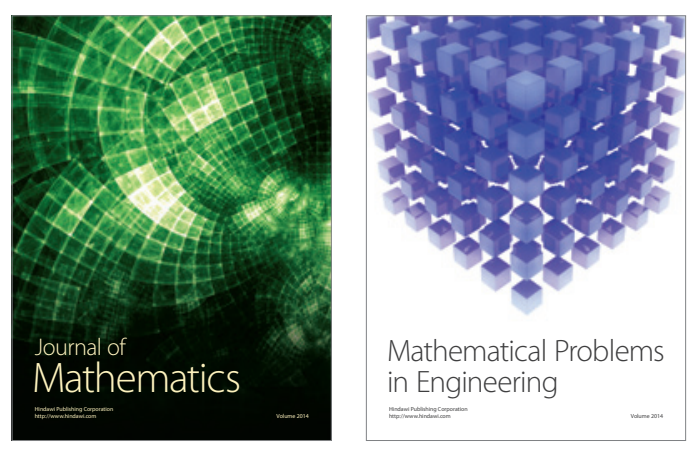

Mathematical Problems in Engineering
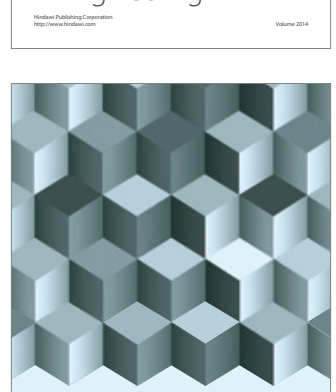

Journal of

Function Spaces
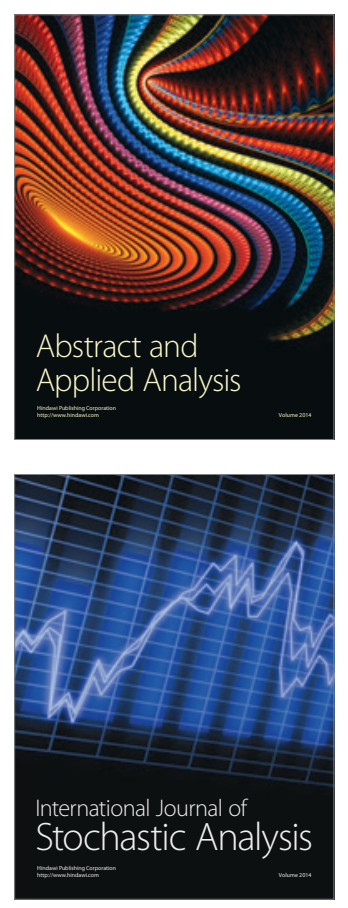

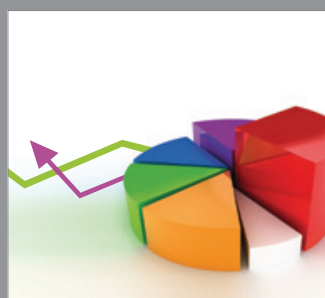

ournal of

Probability and Statistics

Promensencen
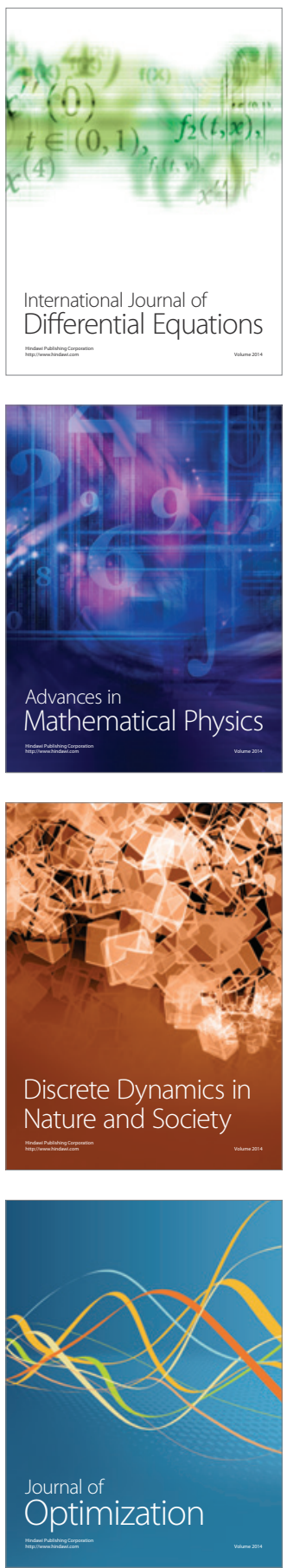